\title{
Hierarchic Analysis of Chaotic Scattering in Reactive Collisions *
}

\author{
Zoltán Kovács**a and Laurent Wiesenfeld ${ }^{\mathrm{b}}$ \\ a Inst. for Theoretical Physics, Univ. of Amsterdam, Valckenierstraat 65, NL-1018 XE Amsterdam. \\ b Laboratoire de Spectrométrie Physique, Université Joseph-Fourier-Grenoble, BP 87, \\ F-38402 Saint-Martin-d'Hères Cédex
}

\section{Z. Naturforsch. 49a, 1229-1232 (1994); received October 12, 1994}

The method of classical trajectories is applied for a double Morse potential modeling indirect reactive collisions of three collinear atoms. The system shows chaotic scattering, which is studied in details through a hierarchic analysis of the singularities of the time delay function. Tools of the thermodynamic formalism are used to extract global quantities characterizing the scattering process.

\section{The Model}

Our aim is to describe some properties of chemical reactions of the type $\mathrm{A}+\mathrm{BC} \leftrightarrow \mathrm{ABC} \leftrightarrow \mathrm{AB}+\mathrm{C}$ (e.g., $\mathrm{CO}+\mathrm{N} \leftrightarrow \mathrm{CNO} \leftrightarrow \mathrm{CN}+\mathrm{O}$ ). We consider the classical motion of the nuclei in a smooth potential surface (a consequence of the Born-Oppenheimer approximation), assuming also a collinear configuration (Fig. 1)

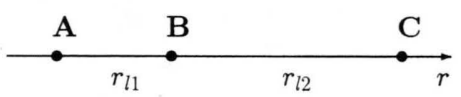

Fig. 1. The collinear configuration with local coordinates $r_{11}$ and $r_{12}$.

and neglecting interaction between the atoms $\mathrm{A}$ and C. By introducing relative (local) coordinates $r_{11}=\left|r_{\mathrm{A}}-r_{\mathrm{B}}\right|, r_{12}=\left|r_{\mathrm{C}}-r_{\mathrm{B}}\right|$ and their conjugate momenta $p_{11}, p_{12}$, and by eliminating center-of-mass motion, we obtain a Hamiltonian of two oscillators $\mathrm{AB}$ and $\mathrm{BC}$ with kinetic coupling:

$$
\begin{aligned}
\mathscr{H}=\frac{p_{11}^{2}}{2 m_{\mathrm{AB}}} & +\frac{p_{12}^{2}}{2 m_{\mathrm{CB}}}-\frac{1}{m_{\mathrm{B}}} p_{11} p_{12}+V_{\mathrm{AB}}\left(r_{11}\right) \\
& +V_{\mathrm{BC}}\left(r_{12}\right),
\end{aligned}
$$

$m_{\mathrm{AB}}, m_{\mathrm{CB}}$ being reduced masses. To simplify the model further, we consider the symmetric, or ABA, case with $V$ taken as a Morse potential: $V(r)=D\left(1-e^{-a\left(r-R_{\mathrm{e}}\right)}\right)^{2}$. The parameters $a, D$, and $m_{\mathrm{AB}}$ can be used to introduce dimensionless variables $r_{1}, r_{2}$ in the problem.

* Presented at the Workshop on Thermodynamic Formalism, Lavin (Engadin), Switzerland, April 23-26, 1994

** On leave from the Institute for Theoretical Physics, Eötvös University, Budapest, also at ${ }^{\mathrm{b}}$.

Reprint requests to Dr. Z. Kovács.
In this picture, the motion of a point particle representing the state of our system is not a pure potential motion. In order to restore that, we change from kinetic to potential coupling by introducing oblique coordinates. In the ABA case this can be done by introducing the angle $\phi$ (between 0 and $\pi / 2$ ) through the relation $\cos \phi=1 /\left(1+m_{\mathrm{B}} / m_{\mathrm{A}}\right)$. Then the oblique coordinates are defined by $q_{1}=r_{1}+r_{2} \cos \phi$ and $q_{2}=r_{2} \sin \phi$, and the formulas for the new momenta $p_{1}$ and $p_{2}$ follow easily. In these variables, the (dimensionless) Hamiltonian now takes the form

$$
\begin{aligned}
\mathscr{H}= & \frac{\sin ^{2} \phi}{2}\left(p_{1}^{2}+p_{2}^{2}\right)+\frac{1}{2}\left[1-e^{-\left(q_{1}-q_{2} \cot \phi-r_{\mathrm{e}}\right)}\right]^{2} \\
& +\frac{1}{2}\left[1-e^{-\left(q_{2} / \sin \phi-r_{\mathrm{e}}\right)}\right]^{2} .
\end{aligned}
$$

The overall topology in phase space is determined by two scaled parameters only: the mass ratio $m_{\mathrm{B}} / m_{\mathrm{A}}$, through the angle $\phi$, and the quantity $\widetilde{E}=E / D$, where $E$ is the total energy of the system. The motion is bounded for $\tilde{E}<1$ and can be chaotic at not too small energies. The interesting region is $1<\widetilde{E}<2$ when the motion of one atom can become unbounded. As can be seen from (2), the potential surface is mirror symmetric with respect to the line $q_{2}=q_{1} \tan (\phi / 2)$ and has two channels, parallel to the $q_{1}$ axis and the line $q_{2}=q_{1} \tan \phi$, that correspond to the two different outcomes of the collision. In the following, we fix the value of $\phi$ by choosing a mass ratio $m_{\mathrm{B}} / m_{\mathrm{A}}=2$.

\section{Chaotic Scattering in the Model}

For $\tilde{E}>1$, two periodic orbits appear sitting at the ends of the channels characterized by the independent oscillatory behaviour of an $\mathrm{AB}$ pair and the asymptot- 

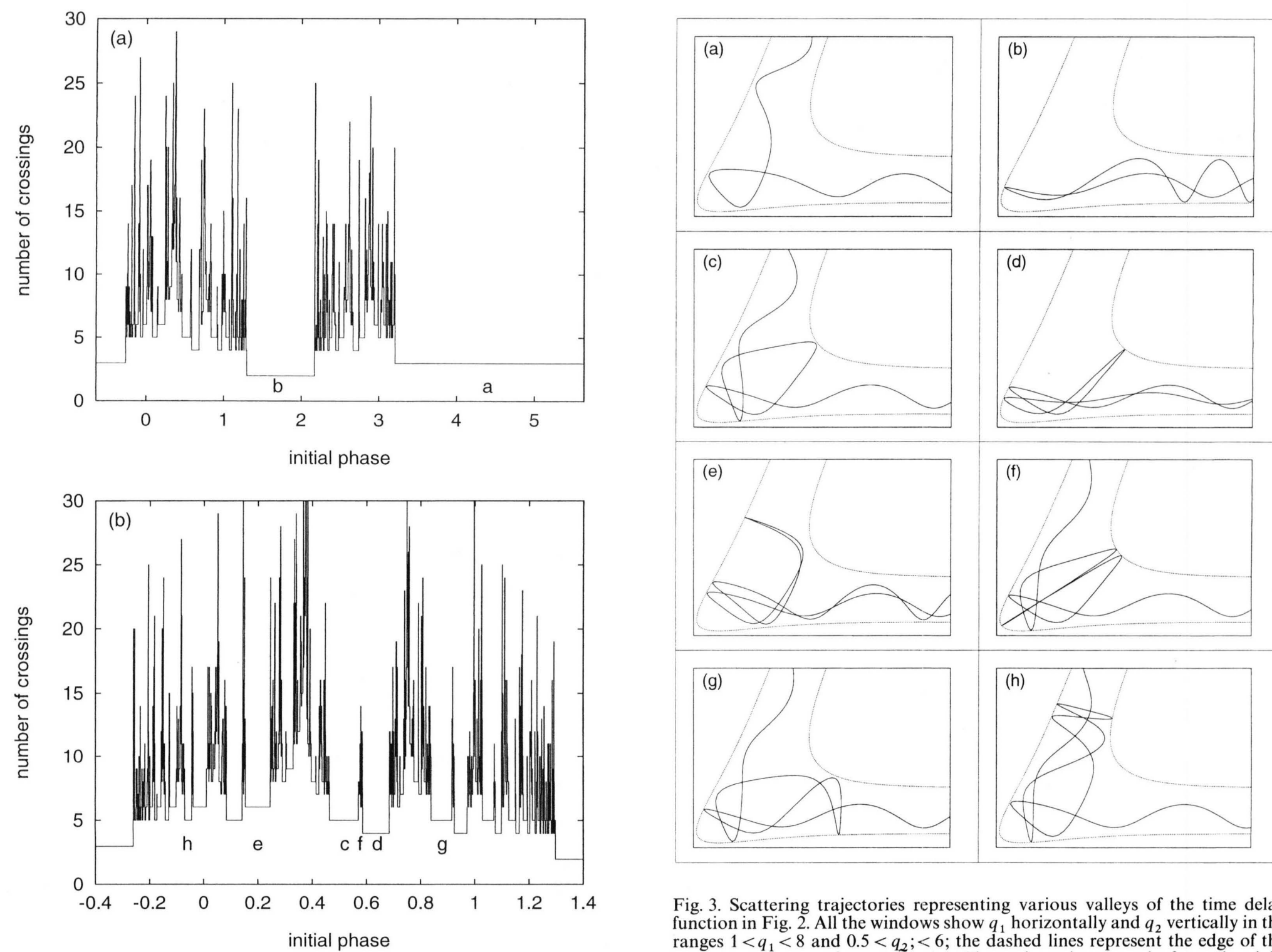

Fig. 3. Scattering trajectories representing various valleys of the time delay function in Fig. 2. All the windows show $q_{1}$ horizontally and $q_{2}$ vertically in the ranges $1<q_{1}<8$ and $0.5<q_{2} ;<6$; the dashed lines represent the edge of the constant energy surface at $\widetilde{E}=1.4$. The trajectories come in from the right along the horizontal channel and are characterized by the number of loops $n$ performed in the central region. From those shown here (a) and (b) correspond

Fig. 2. (a) The discrete time delay function for $\widetilde{E}=1.4$ (b) Blowup of the left block of singularities. The $a-h$ under the graphs identify the valleys represented by the corresponding typical trajectories in Fig. 3(a)-(h), respectively. 
ically free translation of a single A atom. These infinitely distant orbits imply the possibly of scattering orbits starting from an asymptotic free motion of an $\mathrm{A}$ atom toward the oscillating $\mathrm{AB}$ molecule (incoming part) and ending in a single $A$ atom running away from the remaining $\mathrm{AB}$ pair to infinity (outgoing part). The asymptotic parts are connected via an irregular behaviour around the center of the potential. The scattering orbits thus show a chaotic behaviour on a finite time scale (transient chaos), leading to chaotic scattering [1], characterized by the extreme sensitivity of the outcome of scattering on the initial conditions.

Most of the unstable bounded orbits that exist below $\widetilde{E}=1$ remain bounded for $\widetilde{E}>1$ too and form a non-attractive chaotic set [2] in phase space. The stable manifolds of these orbits extend smoothly to infinity making possible for scattering trajectories to get close to and following them for a while. It is exactly this property that causes the chaotic features in the scattering. These features are also manifested in the irregular behaviour of the time delay function measuring scattering time as a function of the initial condition $[2,3]$. Figure 2 shows, how the number of crossings of a trajectory with the symmetry axis of the potential - a discretized time delay - varies as a function of the initial phase of the oscillator (the trajectories are started at the end of the horizontal channel with a fixed incoming translational momentum). It possesses singularities arranged in a fractal pattern. The singularities of the time delay function correspond to initial conditions that sit on the stable manifolds of the chaotic set. Therefore, the pattern of the singularities reflects the fractal structure of the chaotic set itself. Thus the analysis of the singularity structure in the time delay function allows us to obtain important quantities characterizing the chaotic set.

\section{A Hierarchic Analysis}

The time delay function contains smooth parts (valleys), corresponding to simple scattering trajectories, separated by irregular regions that show, in a blowup, many smaller valleys. The topological properties of the scattering orbits are the same inside a valley but change when looking into another valley; moreover, in general, the narrower valleys contain more complex orbits (Figure 3). This suggest a hierarchic approach [4]: we identify basic blocks in the time delay function by deleting the valleys of the simplest orbits, then

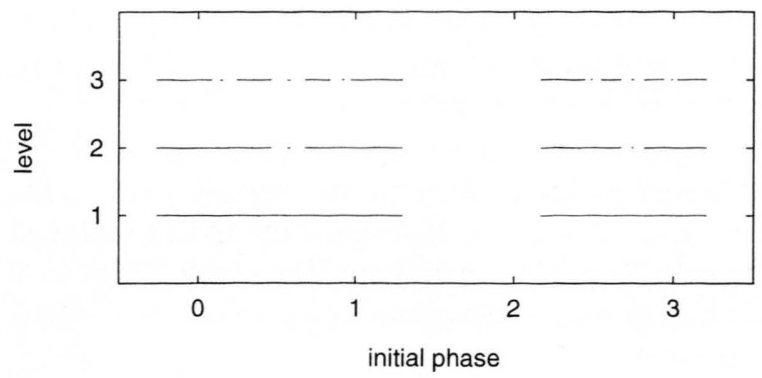

Fig. 4. The first 3 levels of the hierarchy found in Figure 2. The intervals at level $n$ correspond to initial conditions that lead to trajectories performing at least $n$ loops before their final exit loops. Each interval splits into three parts one level higher in accordance with the ternary hierarchy (the splitting of the smallest intervals at level 2 is invisible at this printing resolution). The lengths of these intervals are used in the thermodynamic analysis as the basis of a quantitative description.

refine these blocks one by one by locating and removing the valleys of the simplest orbits inside the new blocks. This procedure can be repeated infinitely many times due to the fractal structure and provides us with a hierarchy of smaller and smaller blocks covering the irregular part of the time delay function.

Figure 4 shows the first few levels of the hierarchy found for Fig. 2: the blocks of level $n$ contain orbits performing at least $n$ loops before their exit loop in the central region of the potential. This reveals a ternary organization: escaping trajectories inside a block can leave along one of the two channels, thus leaving three new smaller blocks of orbits that continue their wandering around the center. The details of the hierarchy depend on the energy and the mass ratio but are expected not to change in large domains of the parameter pace. For example, the same ternary structure appears for $\widetilde{E}=1.2$ and 1.4 . The ternary organization is the consequence of the topology of the potential surface, therefore it is expected to show up for other models with similar topological properties.

\section{Scaling Properties}

The hierarchic data provided by the analysis of the time delay function can be used to extract important quantities characterizing the chaotic scattering process and the self-similar properties of its chaotic set [3]. A quantity of central interest is the free energy $F(\beta)$ introduced in the spirit of the thermodynamic formal- 
ism of dynamical systems [5] via the relation

$$
\sum_{i}\left(l_{i}^{(n)}\right)^{\beta} \sim e^{-\beta F(\beta) n},
$$

where $\beta$ is an arbitrary real number and $n \gg 1$. $l_{i}^{(n)}$ denote the lengths of parameter intervals $I_{i}^{(n)}$ along the initial condition axis that cover the blocks sitting in the $n$th level of the hierarchy. The graph $\beta F(\beta)$ vs. $\beta$ is monotonically increasing with a nonpositive second derivative.

Several values of $\beta F(\beta)$ yield basic characteristics of the scattering process. The escape rate $\chi$ describes the exponential decay of the probability $P_{n}$ for a scattering trajectory to survive through $n$ loops in the scattering center: $P_{n} \sim e^{-x n}$ (for large $n$ ) which is proportional to the sum of the lengths of all intervals at the $n$-th level, thus $\varkappa=F(1)$. The topological entropy $K_{0}$ is defined as the quantity characterizing the exponential growth of the number $N(n)$ of intervals $I_{i}^{(n)}$ with the level index $n$. Therefore we obtain it as $K_{0}=-\left.(\beta F(\beta))\right|_{\beta=0}$. It also appears as the exponential growth rate of the number of smooth pieces (valleys) in the time delay function. The average Lyapunov exponent $\bar{\lambda}$ of the dynamics on the chaotic invariant set and the fractal dimension $D_{0}$ of the singularities in the time delay function can be calculated as $\bar{\lambda}=\left.(\beta F(\beta))^{\prime}\right|_{\beta=1}$ and $F\left(D_{0}\right)=0$. For $\tilde{E}=1.2$ and 1.4 , we obtained through this procedure the results listed in Table 1.

The value of $K_{0}$ reflects the ternary hierarchy, while the other quantities give a measure of chaoticity in the scattering process. In addition, $1 / \varkappa$ multiplied by the average time taken for a loop can be regarded as an estimate of the lifetime of the intermediate ABA complex in the reaction. Other relevant average quantities
Table 1. Global quantitative characteristics of chaotic scattering in the double Morse potential. Values given below are extracted from free energy $\beta F(\beta)$. Uncertainties are of the order of $10 \%$.

\begin{tabular}{lll}
\hline Quantity & Value for & Value for \\
& $\widetilde{E}=1.2$ & $\widetilde{E}=1.4$ \\
\hline$K_{0}$ & $\ln 3$ & $\ln 3$ \\
$D_{0}$ & 0.85 & 0.77 \\
$\lambda$ & 0.83 & 0.88 \\
$x$ & 0.12 & 0.22 \\
\hline
\end{tabular}

can also be calculated by similar hierarchic approaches. This analysis is not restricted to the double Morse potential: it is expected to work for a large number of other classical model potentials used in chemical reactivity studies.

\section{Acknowledgements}

We thank A. Driss of the careful derivations of relevant Hamiltonians and T. Tél for insightful discussions. The present study has been made possible through an EEC grant PECO CIPA 3511. One of the authors (Z.K.) is grateful for the kind hospitality of Laboratoire Spectrométrique Physique, and acknowledges partial financial support from the Hungarian Scientific Research Foundation (grant numbers: OTKA 2090 and F 4286) and the PHARE ACCORD Program (OMFB No. H9112-0378). This work has been completed under a research project financed by the Foundation for Fundamental Research on Matter in The Netherlands (project number: 93 BR 1099).
[1] For a review, see E. Ott and T. Tél, Chaos 3, 417 (1993) and references therein.

[2] S. Bleher, E. Ott, and C. Grebogi, Phys. Rev. Lett. 63, 919 (1989); S. Bleher, C. Grebogi, and E. Ott, Physica D46, 87 (1990).

[3] Z. Kovács and T. Tél, Phys. Rev. Lett. 64, 1617 (1990); T. Tél, Phys. Rev. A 44, 1034 (1991).
[4] Z. Kovács and L. Wiesenfeld, Chaotic scattering in reactive collisions: A classical analysis, reprint, 1994.

[5] D. Ruelle, Thermodynamic Formalism (Addison-Wesley, Reading, 1978); M. J. Feigenbaum, M. H. Jensen, and I. Procaccia, Phys. Rev. Lett. 56, 1503 (1986); T. Bohr and D. Rand, Physica D 25, 387 (1987). 